\title{
The Solvability of Probabilistic Regresses. A Reply to Frederik Herzberg
}

\author{
David Atkinson and Jeanne Peijnenburg
}

\begin{abstract}
We have earlier shown by construction that a proposition can have a well-defined nonzero probability, even if it is justified by an infinite probabilistic regress. We thought this to be an adequate rebuttal of foundationalist claims that probabilistic regresses must lead either to an indeterminate, or to a determinate but zero probability. In a comment, Frederik Herzberg has argued that our counterexamples are of a special kind, being what he calls 'solvable'. In the present reaction we investigate what Herzberg means by solvability. We discuss the advantages and disadvantages of making solvability a sine qua non, and we ventilate our misgivings about Herzberg's suggestion that the notion of solvability might help the foundationalist.

We further show that the canonical series arising from an infinite chain of conditional probabilities always converges, and also that the sum is equal to the required unconditional probability if a certain infinite product of conditional probabilities vanishes.
\end{abstract}

Keywords

Probabilistic justification, regress problem, foundationalism, infinitism.

Published in Studia Logica, 94 (2010) 347-353.

According to classical foundationalism, an infinite probabilistic regress either yields zero or remains indeterminate. In several papers we have given counterexamples to this claim. That is, we have demonstrated that a proposition can have a well-defined nonzero probability, even though its probabilistic justification is forever postponed [1], [2], [3], [4].

In an interesting comment involving a pretty use of nonstandard analysis, Frederik Herzberg [5] has proved that our counterexamples are consistent, in the sense that they have a model. However, he also argues that they are of a very special kind. For our counterexamples are what he calls 'solvable' and most consistent probabilistic regresses lack that property. Our counterexamples are thus exceptional, and this leads Herzberg to conclude that 
there is a way in which a foundationalist might defend herself. For she might now argue that generically we are unable to calculate or estimate an infinite probabilistic regress explicitly. In other words, she might concede that some propositions which are justified by a probabilistic regress can have a well-defined nonzero probability, but still deny that this is generally the case.

In the present reply to Herzberg we will focus on the notion of solvability. (i) We will first investigate what Herzberg means by 'solvable'. (ii) Then we discuss the pros and cons of the requirement that a probabilistic regress can justify a proposition only if that regress is in fact solvable. (iii) Finally, we consider two alternative ways in which a foundationalist might defend herself.

(i) What does it mean to say that a probabilistic regress is solvable? Herzberg writes: "A probabilistic regress $\langle\alpha, \beta\rangle$ is solvable if for any model $\langle P, S\rangle$ of $\langle\alpha, \beta\rangle$ one can derive a closed-form expression for $P\left(S_{0}\right)$." $\quad[5$, p. 3; for the notation, see Herzberg's Definition 2]. However, he also notes that "[i]t is difficult to provide a precise definition of solvability, since the notion of a closed-form expression is not a well-defined mathematical concept." (ibid.) In the first footnote of his paper Herzberg suggests that closed-form expressions include rational numbers, but that it is unclear whether some irrational, algebraic or transcendental numbers also fall under this heading. "All one can say", he writes, "is that in all of these cases, any candidate for [a] set of closed-form expressions will be a countable set $C$ of real numbers." (ibid.) Acknowledging that there is no agreement on what this set $C$ looks like, he concludes with a counterfactual: "if there were universal agreement on the set $C$ of closed-form expressions of real numbers, a candidate for a definition of closed-form solvability would be as follows" (ibid., pp. 3-4) after which he defines closed-form solvability in terms of $C$.

In order to gain a better understanding of solvability, let us take a closer look at the structure of our counterexamples. The typical form of these counterexamples is:

$$
P\left(S_{0}\right)=A+B
$$

where $P\left(S_{0}\right)$ is the probability associated with the target proposition $S_{0}, A$ is an infinite series of conditional probabilities only, and $B$ is a remainder term, containing precisely one unconditional probability as a factor:

$$
\begin{aligned}
A & =\lim _{s \rightarrow \infty}\left\{\beta_{0}+\gamma_{0} \beta_{1}+\gamma_{0} \gamma_{1} \beta_{2}+\ldots+\gamma_{0} \gamma_{1} \ldots \gamma_{s-1} \beta_{s}\right\} \\
B & =\lim _{s \rightarrow \infty}\left\{\gamma_{0} \gamma_{1} \ldots \gamma_{s} P\left(S_{s+1}\right)\right\}
\end{aligned}
$$


(for the meaning of the symbols $\beta$ and $\gamma$ see Herzberg's [5] and our [2]).

Following Herzberg, we shall say that Eq.(1) is solvable if and only if we can write down a closed-form expression for the sum $A+B$, for example an expression in terms of a finite number of rational terms. Now it can be proved that the infinite series of conditional probabilities $A$ always converges, so $A$ certainly is equal to a unique and well-defined number (for a proof, see Appendix A). Moreover, in the usual cases $B$ will tend to zero (see Appendix B). This means that, as a rule, the infinite regress of conditional probabilities produces a convergent series that is equal to the unconditional probability of the target proposition $S_{0}$. In other words: as a rule the unique and welldefined value of $A$ coincides with $P\left(S_{0}\right)$.

From this it does not follow, however, that this unique value can actually be written down as a closed-form expression consisting only of known functions. It might after all be an expression outside Herzberg's set $C$. In general the structure $P\left(S_{0}\right)=A+B$ is solvable if we can write the unique value of $A$ in an acceptable closed form. Since mostly we are not able to do this, we agree with Herzberg that most convergent series are not solvable in his sense. The generic case is indeed one in which an infinite probabilistic regress yields a convergent series, the sum of which cannot be written down in a closed form based on familiar functions.

(ii) In Herzberg's view, this might help the foundationalist. For she can now argue that most probabilistic regresses are unsolvable and thus that our solvable counterexamples form an exceptional class. At the end of his paper Herzberg concludes that the generic, unsolvable probabilistic regresses could produce a very small number: "for a given regress, $P\left(S_{0}\right)$ might be very close to zero" [5, last sentence]. He thus suggests that, while the solvable regresses yield a non-zero value for $P\left(S_{0}\right)$, the unsolvable ones might confer on this probability uncontrollably tiny values. But this suggestion could easily mislead the reader. As we see from Eqs. (1)-(2), the value of $P\left(S_{0}\right)$ cannot be smaller than $\beta_{0}$. Whenever the latter is not very close to zero, $P\left(S_{0}\right)$ will not be close to zero either. Since this goes for solvable and unsolvable regresses alike, most of the generic, unsolvable regresses will thus not end up close to zero, much as in the case of nongeneric, solvable regresses.

A foundationalist might react to this by banning unsolvable regresses altogether. Indeed, if she is a rigid constructivist, she would doubt the very existence of such regresses, so she will certainly not acknowledge them as potential counterexamples in our sense. 
How reasonable is the position of this constructive foundationalist? In order to answer this question, we should bear in mind that invoking solvability carries with it two moments of choice. First, we must choose a definition of solvability, which in this case amounts to specifying Herzberg's set $C$. Which expressions are we supposed to include and which are we supposed to leave out? As Herzberg notes, there is no clear answer to this question. Second, and independently of what solvability looks like, we must decide whether or not we require counterexamples to be solvable. A rigid constructivist, as we have seen, will indeed require solvability as a sine qua non. For her, a probabilistic regress justifies a proposition only if its sum can be written as a closed-form expression. We are however inclined to take a laxer stance. It is true that most of our counterexamples yield rational numbers and thus are solvable in Herzberg's sense. But this is only an accidental feature, introduced for reasons of simplicity and immediate intelligibility. We never meant solvability to be an essential characteristic of our counterexamples.

Of course, demanding that only solvable series can be counterexamples has the advantage of ensuring that the latter are easily calculable. But the demand has obvious drawbacks as well. If interpreted narrowly, it would exclude the use of many known and unknown higher functions. Isn't it standard practice to define transcendental numbers and functions by means of convergent series, by integral transforms, or by other unsolvable, but calculable expressions? And are there not infinitely many other new transforms that can be computed? Personally we see no good reason to ban any of them; but this is after all more a matter of taste than a matter of fact.

(iii) Assuming that a constructivist approach does not really help our hypothetical foundationalist, what other lifelines are available to her? We can think of two. The infinite product $\gamma_{0} \gamma_{1} \gamma_{2} \ldots$ normally diverges to zero (see Appendix B), so that the term $B$ in Eq.(1) vanishes. However - and this is the first lifeline - a foundationalist might wish to claim that a "prime mover' $\left(S_{s+1}\right.$ in the limit $s \rightarrow \infty$, thus infinitely far away) is still required to bring the whole epistemic chain into existence, even though the stochastic support that it gives to $S_{0}$ has shrivelled away to nothing. If she were to grasp this lifeline, the foundationalist would commit herself to a position that we might call 'epistemological deism'. Seventeenth and eighteenth century deists preached that God created the world, including the laws of nature, but waived any interference after that. In a similar vein, our counterexamples postulate an infinitely remote beginning of the justificatory regress, but deny 
that this beginning has any influence whatsoever on the probability value of the target proposition. All justification for the target proposition stems from the conditional probabilities that make up the infinite series $A$.

Any foundationalist who feels uncomfortable with this meagre option might choose the second lifeline. She could seek solace in the atypical case where the infinite product $\gamma_{0} \gamma_{1} \gamma_{2} \ldots$ (and thus $B$ ) converges to a value that is not zero. The general expression (1) for the required probability leads to

$$
P\left(S_{0}\right)=\beta_{0}+\sum_{n=1}^{\infty} \beta_{n} \prod_{s=0}^{n-1} \gamma_{s}+P^{*} \prod_{n=0}^{\infty} \gamma_{n},
$$

where

$$
P^{*}=\lim _{n \rightarrow \infty} P\left(S_{n}\right),
$$

on condition that this limit exists. In order to calculate $P\left(S_{0}\right)$ we need now not only the conditional probabilities, but also $P^{*}$, the unconditional probability associated with an infinitely distant prime mover; for the latter still exerts probabilistic influence on the value of $P\left(S_{0}\right)$.

However, the second lifeline comes with a price tag. For not only is this class atypical, it is also characterized by a sequence, $\left\{\gamma_{n}\right\}$, that tends very rapidly to unity as $n$ tends to infinity. This means that $\beta_{n}$ tends very rapidly to zero, and $\alpha_{n}=\beta_{n}+\gamma_{n}$ tends very rapidly to one. If it were the case that $\alpha_{n}=1$ and $\beta_{n}=0$ exactly for some $n$, then the relation between $S_{n}$ and $S_{n+1}$ would be one of bi-implication, $S_{n} \longleftrightarrow S_{n+1}$, thus guaranteeing that $S_{n}$ and $S_{n+1}$ have the same truth values. Stochastically speaking, the link between the two propositions has been short-circuited, and the same would apply to any link for which the corresponding $\gamma_{n}$ is precisely equal to one. If however $\gamma_{n}$ is not precisely equal to one, but tends asymptotically very quickly to that value, we might dub the relation between successive propositions in the epistemic chain, at any rate for sufficiently large values of $n$, one of quasibi-implication. For small values of $n$ the values of $\gamma_{n}$ might depart radically from unity, so we have the finite beginnings of a normal epistemic chain. The infinite tail, however, would be one of quasi-bi-implication. In other words, all but a finite part of the chain is approximately short-circuited through to the ground. The probabilistic chain might be called quasi-finite, and it could perhaps serve as a generalization of the standard foundationalist requirement that the series of justification be finite. However, this option seems little more than a simple reformulation of the foundationalist stance and thus appears 
to come close to begging the question of whether justification by probabilistic regress is possible.

\section{Appendix A. Proof that the series converges}

The unconditional probabilities $P\left(S_{n}\right)$ and $P\left(S_{n+1}\right)$ are related by the rule of total probability,

$$
P\left(S_{n}\right)=\beta_{n}+\gamma_{n} P\left(S_{n+1}\right)
$$

where

$$
\beta_{n}=P\left(S_{n} \mid \neg S_{n+1}\right) \quad \text { and } \quad \gamma_{n}=P\left(S_{n} \mid S_{n+1}\right)-P\left(S_{n} \mid \neg S_{n+1}\right),
$$

with $\gamma_{n}>0$ for all $n$, which is the condition of probabilistic support. Eq.(3) can be iterated from $n=0$ up to $n=s$, with the result ${ }^{1}$

$$
P\left(S_{0}\right)=\beta_{0}+\sum_{n=1}^{s} \gamma_{0} \gamma_{1} \ldots \gamma_{n-1} \beta_{n}+\gamma_{0} \gamma_{1} \ldots \gamma_{s} P\left(S_{s+1}\right) .
$$

In the limit that one lets $s$ go to infinity, the resulting infinite series is in fact convergent, as we will now prove. Since $\beta_{n}+\gamma_{n}=P\left(S_{n} \mid S_{n+1}\right) \leq 1$, it follows that $\beta_{n} \leq 1-\gamma_{n}$, and so

$$
\begin{aligned}
& \sum_{n=1}^{s} \gamma_{0} \gamma_{1} \ldots \gamma_{n-1} \beta_{n} \leq \sum_{n=1}^{s} \gamma_{0} \gamma_{1} \ldots \gamma_{n-1}\left(1-\gamma_{n}\right) \\
& \quad=\left(\gamma_{0}-\gamma_{0} \gamma_{1}\right)+\left(\gamma_{0} \gamma_{1}-\gamma_{0} \gamma_{1} \gamma_{2}\right)+\ldots+\left(\gamma_{0} \gamma_{1} \ldots \gamma_{s-1}-\gamma_{0} \gamma_{1} \ldots \gamma_{s}\right) \\
& \quad=\gamma_{0}-\gamma_{0} \gamma_{1} \ldots \gamma_{s} \leq \gamma_{0}
\end{aligned}
$$

which is finite and independent of $s$, and therefore also valid in the limit that $s$ is taken to infinity. Since all the $\beta_{n}$ are nonnegative and all the $\gamma_{n}$ are positive, it follows that the infinite series of positive terms

$$
A=\beta_{0}+\sum_{n=1}^{\infty} \gamma_{0} \gamma_{1} \ldots \gamma_{n-1} \beta_{n}
$$

${ }^{1}$ Formula (5) can be formally proved by mathematical induction as follows:

1. Since $P\left(S_{0}\right)=\beta_{0}+\gamma_{0} P\left(S_{1}\right)$, Eq.(5) is clearly true when $s=0$.

2. Suppose $P\left(S_{0}\right)=\beta_{0}+\sum_{n=1}^{s-1} \gamma_{0} \gamma_{1} \ldots \gamma_{n-1} \beta_{n}+\gamma_{0} \gamma_{1} \ldots \gamma_{s-1} P\left(S_{s}\right)$ for some $s$. Then, on substituting $\beta_{s}+\gamma_{s} P\left(S_{s+1}\right)$ for $P\left(S_{s}\right)$ in this formula, we obtain the right-hand side of Eq.(5).

Therefore, if (5) is true for some $s$, it is true for any larger value of $s$. But it is true for $s=0$, so it is true for $s=1,2,3 \ldots$. 
is convergent, for the partial sums (6) are bounded by $\gamma_{0}$.

\section{Appendix B. When does the remainder term vanish?}

Consider the remainder term in Eq. (5), namely $\gamma_{0} \gamma_{1} \ldots \gamma_{s} P\left(S_{s+1}\right)$. We will show that the coefficient in front of $P\left(S_{s+1}\right)$, i.e. $\gamma_{0} \gamma_{1} \ldots \gamma_{s}$, usually tends to zero in the infinite $s$ limit. Since $\gamma_{n}=\exp \left[\log \gamma_{n}\right]=\exp \left[-\left|\log \gamma_{n}\right|\right]$,

$$
\gamma_{0} \gamma_{1} \gamma_{2} \gamma_{3} \ldots=\prod_{n=0}^{\infty} \gamma_{n}=\exp \left[-\sum_{n=0}^{\infty}\left|\log \gamma_{n}\right|\right] \text {. }
$$

So $\gamma_{0} \gamma_{1} \ldots \gamma_{s}$ has the limit zero as $s$ goes to infinity if and only if the sum

$$
\sum_{n=0}^{\infty}\left|\log \gamma_{n}\right|
$$

diverges. Since all the terms in the series (8) are positive, the series can only converge, or diverge to $+\infty$. Unless $\gamma_{n}$ tends very quickly to 1 , the series will indeed diverge; hence an infinite product of terms, each of which is smaller than one, is usually equal to zero. In this case

$$
B=\lim _{s \rightarrow \infty}\left\{\gamma_{0} \gamma_{1} \ldots \gamma_{s} P\left(S_{s+1}\right)\right\}
$$

is also zero, since $P\left(S_{s+1}\right)$ is bounded by unity.

Exceptionally, if $\gamma_{n}$ tends rapidly to 1, this will not be the case. For example, if

$$
1-\gamma_{n} \sim n^{-a} \text { as } n \rightarrow \infty,
$$

then the sum (8) converges if $a>1$, but it diverges if $0<a \leq 1$. If $\gamma_{n}$ tends less quickly still to one, or does not tend to unity at all, which may be regarded as 'usual', the series is divergent, and by convention one says that the corresponding infinite product (7) diverges to zero.

\section{Acknowledgement}

We should like to thank Frederik Herzberg for his comments on an earlier version of this paper, which led to a more nuanced discussion of solvability. 


\section{References}

[1] Peijnenburg, J., 'Infinitism Regained', Mind, 116:597-602, 2007.

[2] Peijnenburg, J., and D. Atkinson, 'Probabilistic Justification and the Regress Problem', Studia Logica 89(3):333-341, 2008.

[3] Atkinson, D., and J. Peijnenburg, 'Justification by an Infinity of Conditional Probabilities', Notre Dame Journal of Formal Logic 50:183193, 2009.

[4] Peijnenburg, J., and D. Atkinson, 'Grounds and Limits. Reichenbach and Foundationalist Epistemology', Synthese, 181 113-124, 2011.

[5] Herzberg, F., 'The Consistency of Probabilistic Regresses. A Reply to Jeanne Peijnenburg and David Atkinson', Studia Logica, this number.

David Atkinson and Jeanne Peijnenburg Faculty of Philosophy University of Groningen Oude Boteringestraat 52

9712 GL Groningen, Netherlands d.atkinson@rug.nl jeanne.peijnenburg@rug.nl 